\title{
On the Autocorrelation Properties of Long-Memory GARCH Processes*
}

\author{
Menelaos Karanasos ${ }^{\mathrm{a}}$, Zacharias Psaradakis ${ }^{\mathrm{b}}$ and Martin Sola ${ }^{\mathrm{b}, \mathrm{c}}$

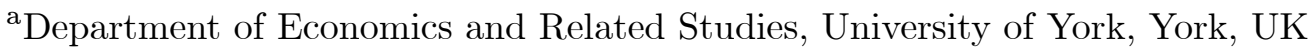 \\ ${ }^{\mathrm{b}}$ School of Economics, Mathematics \& Statistics, Birkbeck College, London, UK \\ ${ }^{\mathrm{c}}$ Department of Economics, Universidad Torcuato Di Tella, Buenos Aires, Argentina
}

First Draft: October 2001; This Version: May 2002

\begin{abstract}
This paper derives the autocorrelation function of the squared values of long-memory GARCH processes. The latter are of much interest since they can produce the long-memory conditional heteroscedasticity that many high-frequency financial time series exhibit. An empirical application illustrating the practical use of our results is also discussed.
\end{abstract}

Keywords: Autocorrelation function; Fractionally integrated GARCH process; Long-memory GARCH process.

\section{Introduction}

A common finding in much of the empirical literature on the second-order structure of highfrequency financial time series is that sample autocorrelations for squared or absolute-valued observations tend to decay very slowly and remain fairly large for long lags (e.g., Dacorogna et al., 1993; Ding et al., 1993; Bollerslev and Mikkelsen, 1996; Ding and Granger, 1996; Breidt et al., 1998). As a consequence, many researchers have proposed extensions of generalized autoregressive conditionally heteroscedastic $(\mathrm{GARCH})$ models which can produce such long-memory behaviour; examples include the models discussed in Robinson (1991), Ding and Granger (1996), Baillie et al. (1996), Bollerslev and Mikkelsen (1996), Robinson and Zaffaroni (1997), and Robinson and Henry (1999), inter alia.

In this paper, we focus on a class of long-memory GARCH (LMGARCH) processes that belong to the family of conditionally heteroscedastic processes introduced by Robinson (1991).

${ }^{*}$ The authors wish to thank Karim Abadir for helpful discussions and an anonymous referee for insightful comments on an earlier draft.

Correspondence to: Zacharias Psaradakis, School of Economics, Mathematics \& Statistics, Birkbeck College, 7-15 Gresse Street, London W1T 1LL, UK; e-mail: z.psaradakis@bbk.ac.uk; fax: +44 2076316416 ; tel.: +44 2076316415 . 
These processes are very closed related to the fractionally integrated GARCH (FIGARCH) processes proposed by Baillie et al. (1996) and share some of the features of fractional ARIMA processes. In particular, shocks to the conditional variance of an LMGARCH process eventually die away to zero (in a forecasting sense), but shock dissipation occurs at a slow hyperbolic rate rather than the faster geometric rate that is characteristic of weakly stationary GARCH processes.

Even though LMGARCH models have become increasingly popular in practice, the statistical properties of time series whose behaviour is governed by such models remain largely unexplored. The present paper is intended as a first step in closing this gap, its contribution being the establishment of convenient representations for the autocorrelation function of the squared values of LMGARCH processes. Such representations are not only of theoretical interest; the practitioner could assess the adequacy of an empirical LMGARCH model by examining whether the qualitatively important features of the correlogram of the squared observations are captured by the autocorrelation function implied by the fitted model.

The remainder of the paper proceeds as follows. Section 2 lays out the models of interest, assumptions and notation. Section 3 presents the autocorrelation functions for squared LMGARCH processes. Section 4 discusses an empirical example. Section 5 concludes.

\section{Long-Memory GARCH Processes}

To establish terminology and notation, recall from Bollerslev (1986) that a $\operatorname{GARCH}(p, q)$ process $\left\{\varepsilon_{t}\right\}$ is defined by the equations

$$
\begin{gathered}
\varepsilon_{t}^{2}=h_{t} \xi_{t}^{2}, \\
B^{\prime}(L) h_{t}=\omega^{\prime}+A^{\prime}(L) \varepsilon_{t}^{2},
\end{gathered}
$$

where $\left\{\xi_{t}, t=0, \pm 1, \pm 2, \ldots\right\}$ are independent and identically distributed random variables with $\mathbb{E}\left(\xi_{t}\right)=\mathbb{E}\left(\xi_{t}^{2}-1\right)=0, A^{\prime}(L) \triangleq \sum_{j=1}^{q} a_{j}^{\prime} L^{j}$, and $B^{\prime}(L) \triangleq 1-\sum_{j=1}^{p} \beta_{j}^{\prime} L^{j}$. (Here and in the sequel, $L$ stands for the lag operator and the symbol ' $\triangleq$ ' is used to indicate equality by definition). It follows that $\left\{\varepsilon_{t}^{2}\right\}$ admits the $\operatorname{ARMA}\left(p^{*}, p\right)$ representation

$$
A^{*}(L) \varepsilon_{t}^{2}=\omega^{\prime}+B^{\prime}(L) v_{t}, \quad v_{t} \triangleq \varepsilon_{t}^{2}-h_{t}
$$

where $A^{*}(L) \triangleq 1-\sum_{j=1}^{p^{*}} a_{j}^{*} L^{j}, p^{*}=\max \{p, q\}, a_{j}^{*}=a_{j}^{\prime}+\beta_{j}^{\prime}\left(j=1, \ldots, p^{*}\right)$, and $\left\{v_{t}\right\}$ is, by construction, a martingale-difference sequence relative to the $\sigma$-field generated by $\left\{\varepsilon_{s}, s \leqslant t\right\}$.

The class of GARCH processes can be generalised by allowing $\left\{\varepsilon_{t}^{2}\right\}$ to satisfy the equation (cf. Robinson, 1991)

$$
\varepsilon_{t}^{2}=\omega+\Omega(L) v_{t},
$$

for some $\omega \in(0, \infty)$ and

$$
\Omega(L) \triangleq \sum_{j=0}^{\infty} \omega_{j} L^{j}, \quad 0<\sum_{j=0}^{\infty} \omega_{j}^{2}<\infty
$$


A strictly stationary $\operatorname{GARCH}(p, q)$ process is a special case of (4) with the coefficients $\left\{\omega_{j}, j \geqslant 0\right\}$ declining towards zero geometrically fast so that $\omega_{j}=O\left(\lambda^{j}\right)$ as $j \rightarrow \infty$ for some $\lambda \in(0,1)$. When $\mathbb{E}\left(\varepsilon_{t}^{4}\right)<\infty$, the geometric decay of $\left\{\omega_{j}, j \geqslant 0\right\}$ implies that the autocorrelations $\left\{\rho_{n}\left(\varepsilon_{t}^{2}\right) \triangleq\right.$ $\left.\operatorname{Corr}\left(\varepsilon_{t+n}^{2}, \varepsilon_{t}^{2}\right), n \geqslant 1\right\}$ are also geometrically decaying. Hence, $\left\{\varepsilon_{t}^{2}\right\}$ exhibits short memory, in the sense that the series $\sum_{n=0}^{\infty} \rho_{n}\left(\varepsilon_{t}^{2}\right)$ is absolutely convergent.

The specification in (4) also includes processes for which the autocorrelations $\left\{\rho_{n}\left(\varepsilon_{t}^{2}\right), n \geqslant 1\right\}$ decay at a rate slower than geometric. One possibility is to allow the coefficients $\left\{\omega_{j}, j \geqslant 0\right\}$ to decay hyperbolically so that $\omega_{j} \sim C j^{-\delta}$ as $j \rightarrow \infty$ for some $\delta \in(1, \infty)$. (Henceforth, $C$ denotes a generic finite positive constant, not necessarily the same throughout, and $a_{n} \sim b_{n}$ as $n \rightarrow \infty$ signifies that $\left.\lim _{n \rightarrow \infty}\left|a_{n}\right| / b_{n}=1\right)$. An important finite parameterization of $\Omega(L)$ that allows for such behaviour is

$$
\Omega(L)=\frac{B(L)}{A(L)(1-L)^{d}},
$$

for some $d \in\left(0, \frac{1}{2}\right)$, with the lag polynomials $A(L) \triangleq 1-\sum_{j=1}^{q} a_{j} L^{j}=\prod_{j=1}^{q}\left(1-\alpha_{j} L\right)$ and $B(L) \triangleq 1-\sum_{j=1}^{p} \beta_{j} L^{j}$ being such that $|A(z)|>0$ and $|B(z)|>0$ for all complex-valued $z$ on the closed unit disk (see, e.g., Robinson and Zaffaroni, 1997; Robinson and Henry, 1999). The fractional-difference operator $(1-L)^{d}$ in (5) is defined as the series

$$
(1-L)^{d} \triangleq F(-d, 1 ; 1 ; L)=\sum_{j=0}^{\infty} \frac{\Gamma(j-d)}{\Gamma(-d) \Gamma(j+1)} L^{j}=\sum_{j=0}^{\infty}\left(\begin{array}{l}
d \\
j
\end{array}\right)(-1)^{j} L^{j}
$$

where

$$
F(a, b ; c ; z) \triangleq \sum_{j=0}^{\infty} \frac{(a)_{j}(b)_{j}}{(c)_{j}} \frac{z^{j}}{j !}
$$

is the Gaussian hypergeometric series, $(b)_{j} \triangleq \prod_{i=0}^{j-1}(b+i)$ is Pochhammer's symbol for the shifted factorial function (with $(b)_{0} \triangleq 1$ ), and $\Gamma(\cdot)$ is the gamma function.

It follows from (4) and (5) that the stochastic volatility $h_{t}$ obeys the equation

$$
h_{t}=\omega \Psi(1)+[1-\Psi(L)] \varepsilon_{t}^{2},
$$

where

$$
\Psi(L) \triangleq 1-\sum_{j=1}^{\infty} \psi_{j} L^{j}=\frac{A(L)(1-L)^{d}}{B(L)},
$$

with $\psi_{j} \geqslant 0(j \geqslant 1)$. Furthermore, since $\operatorname{Var}\left(\varepsilon_{t}^{2}\right)=\mathbb{E}\left(v_{t}^{2}\right) \sum_{j=0}^{\infty} \omega_{j}^{2}$ and $\mathbb{E}\left(v_{t}^{2}\right)=\{1-$ $\left.\left[1 / \mathbb{E}\left(\xi_{t}^{4}\right)\right]\right\} \mathbb{E}\left(\varepsilon_{t}^{4}\right)$ under (4), we have

$$
\mathbb{E}\left(\varepsilon_{t}^{4}\right)=\frac{\omega^{2}}{1-\left\{1-\left[1 / \mathbb{E}\left(\xi_{t}^{4}\right)\right]\right\} \sum_{j=0}^{\infty} \omega_{j}^{2}} .
$$

Hence, square integrability of $\left\{\varepsilon_{t}^{2}\right\}$ requires that $0<\mathbb{E}\left(\xi_{t}^{4}\right)<\infty$ and

$$
\left\{1-\frac{1}{\mathbb{E}\left(\xi_{t}^{4}\right)}\right\} \sum_{j=0}^{\infty} \omega_{j}^{2}<1 .
$$


When, for example, $\xi_{t}$ is normally distributed, this condition becomes $0<\sum_{j=0}^{\infty} \omega_{j}^{2}<\frac{3}{2}$ and we have $\mathbb{E}\left(\varepsilon_{t}^{4}\right)=\omega^{2} /\left(1-\frac{2}{3} \sum_{j=0}^{\infty} \omega_{j}^{2}\right)<\infty$.

Under (4)-(5), the coefficients $\left\{\omega_{j}, j \geqslant 0\right\}$ decay at a slow hyperbolic rate so that $\omega_{j} \sim C j^{d-1}$ as $j \rightarrow \infty$. This in turn implies that the autocorrelations $\left\{\rho_{n}\left(\varepsilon_{t}^{2}\right), n \geqslant 1\right\}$ satisfy

$$
\rho_{n}\left(\varepsilon_{t}^{2}\right)=\frac{\sum_{j=0}^{\infty} \omega_{j} \omega_{j+n}}{\sum_{j=0}^{\infty} \omega_{j}^{2}} \sim C n^{2 d-1} \quad \text { as } n \rightarrow \infty,
$$

provided $\mathbb{E}\left(\varepsilon_{t}^{4}\right)<\infty$. Hence, when the fourth moment of the $\varepsilon_{t}$ exists, $\left\{\varepsilon_{t}^{2}\right\}$ is a weakly stationary process which exhibits long memory for all $d \in\left(0, \frac{1}{2}\right)$, in the sense that the series $\sum_{n=0}^{\infty}\left|\rho_{n}\left(\varepsilon_{t}^{2}\right)\right|$ is properly divergent. For this reason, we shall refer to a process $\left\{\varepsilon_{t}\right\}$ satifying (4) and (5) as an $\operatorname{LMGARCH}(p, d, q)$ process.

A model closely related to the $\operatorname{LMGARCH}(p, d, q)$ specification in (4)-(5) was considered by Baillie et al. (1996), who defined a $\operatorname{FIGARCH}(p, d, q)$ process via the equation

$$
A(L)(1-L)^{d} \varepsilon_{t}^{2}=\omega+B(L) v_{t}
$$

The $\operatorname{FIGARCH}(p, d, q)$ process is strictly stationary and ergodic but not square integrable (see Zaffaroni, 2000). However, since the 'autocorrelations' $\left(\sum_{j=0}^{\infty} \omega_{j} \omega_{j+n}\right) /\left(\sum_{j=0}^{\infty} \omega_{j}^{2}\right)$ in $(7)$ are welldefined even if $\mathbb{E}\left(\varepsilon_{t}^{4}\right)=\infty$ (cf. Henry, 2001), it is not difficult to show that the $\operatorname{FigARCH}(p, d, q)$ and $\operatorname{LMGARCH}(p, d, q)$ processes have the same second-order structure when condition (6) is satisfied.

Finally, it is worth mentioning that Giraitis et al. (2000) have recently studied the properties of infinite-order ARCH processes. Their results, however, do not apply for the specification in (4)-(5) since it does not satisfy the condition $\mathbb{E}\left(\xi_{t}^{2}\right)[1-\Psi(1)]<1$, which was shown to be sufficient for strict stationarity of $\left\{\varepsilon_{t}^{2}\right\}$. The exact properties of processes satisfying (4)-(5) thus remain an open question.

\section{Autocorrelation Structure of LMGARCH Processes}

In this section of the paper, we establish the autocorrelation properties of LMGARCH processes. We begin by considering some low-order processes which have proved to be useful in modelling a variety of financial time series and then proceed to examine the general $\operatorname{LMGARCH}(p, d, q)$ case.

\subsection{LMGARCH( $1, d, 1)$ Process}

The $\operatorname{LMGARCH}(1, d, 1)$ process is defined via the fractional $\operatorname{ARIMA}(1, d, 1)$ equation

$$
\varepsilon_{t}^{2}=\omega+\left(1-\beta_{1} L\right)\left(1-\alpha_{1} L\right)^{-1}(1-L)^{-d} v_{t} .
$$

We begin by giving the infinite moving-average representation of $\varepsilon_{t}^{2}$. 
Lemma 1 The process $\left\{\varepsilon_{t}^{2}\right\}$ admits the infinite moving-average representation

$$
\varepsilon_{t}^{2}=\omega+\sum_{j=0}^{\infty} \omega_{j} v_{t-j}
$$

where

$$
\omega_{j}=\left(\begin{array}{c}
-d \\
j
\end{array}\right)(-1)^{j}+\sum_{k=1}^{j}\left(\begin{array}{c}
-d \\
j-k
\end{array}\right)(-1)^{j-k}\left(\alpha_{1}^{k}-\alpha_{1}^{k-1} \beta_{1}\right) .
$$

Proof. Since

$$
(1-\kappa L)^{-d}=\sum_{j=0}^{\infty}\left(\begin{array}{c}
-d \\
j
\end{array}\right)(-\kappa)^{j} L^{j}
$$

we have

$$
\frac{1-\beta_{1} L}{1-\alpha_{1} L}=\sum_{j=0}^{\infty}\left(\begin{array}{c}
-1 \\
j
\end{array}\right)\left(-\alpha_{1} L\right)^{j}\left(1-\beta_{1} L\right)=1+\sum_{j=1}^{\infty}\left(\alpha_{1}^{j}-\alpha_{1}^{j-1} \beta_{1}\right) L^{j},
$$

and hence (10) follows from (9).

In our first proposition, we obtain the autocorrelation function of $\left\{\varepsilon_{t}^{2}\right\}$.

Proposition 1 The autocorrelation function of $\left\{\varepsilon_{t}^{2}\right\}$ is given by

$$
\rho_{n}\left(\varepsilon_{t}^{2}\right)=\gamma_{n} / \gamma_{0}, \quad n \geqslant 1
$$

where

$$
\begin{aligned}
\gamma_{n}= & \frac{\Gamma(1-2 d)}{\left(1-\alpha_{1}^{2}\right) \Gamma(d) \Gamma(1-d)}\left\{\frac { \Gamma ( d + n ) } { \Gamma ( 1 - d + n ) } \left[\left(1+\beta_{1}^{2}-\beta_{1} \alpha_{1}\right)\right.\right. \\
& \left.\times F\left(d+n, 1 ; 1-d+n ; \alpha_{1}\right)-\alpha_{1} \beta_{1} F\left(d-n, 1 ; 1-d-n ; \alpha_{1}\right)\right] \\
& +\frac{\Gamma(d+n-1)}{\Gamma(n-d)}\left[\alpha_{1}\left(1+\beta_{1}^{2}\right)-\beta_{1}\right] F\left(d-n+1,1 ; 2-d-n ; \alpha_{1}\right) \\
& \left.-\frac{\Gamma(d+n+1)}{\Gamma(2-d+n)} \beta_{1} F\left(d+n+1,1 ; 2-d+n ; \alpha_{1}\right)\right\}, \quad n \geqslant 0 .
\end{aligned}
$$

Proof. On account of (10) and (11) we have

$$
\rho_{n}\left(\varepsilon_{t}^{2}\right)=\frac{\sum_{k=0}^{\infty} \sum_{j=-\infty}^{\infty}\left(\begin{array}{c}
-d \\
|n-j|+k
\end{array}\right)\left(\begin{array}{c}
-d \\
k
\end{array}\right)(-1)^{|n-j|} \sum_{i=0}^{\infty} \pi_{i} \pi_{i+|j|}}{\sum_{k=0}^{\infty} \sum_{j=-\infty}^{\infty}\left(\begin{array}{c}
-d \\
|-j|+k
\end{array}\right)\left(\begin{array}{c}
-d \\
k
\end{array}\right)(-1)^{|-j|} \sum_{i=0}^{\infty} \pi_{i} \pi_{i+|j|}},
$$

where $\pi_{0} \triangleq 1$ and $\pi_{i}=\alpha_{1}^{i}-\alpha_{1}^{i-1} \beta_{1}$ for $i \geqslant 1$. But since

$$
\sum_{k=0}^{\infty}\left(\begin{array}{c}
-d \\
|n-j|+k
\end{array}\right)\left(\begin{array}{c}
-d \\
k
\end{array}\right)(-1)^{|n-j|}=\frac{\Gamma(1-2 d) \Gamma(d+|n-j|)}{\Gamma(d) \Gamma(1-d) \Gamma(1-d+|n-j|)},
$$

it follows that

$$
\rho_{n}\left(\varepsilon_{t}^{2}\right)=\frac{\sum_{j=-\infty}^{\infty} \frac{\Gamma(d+|n-j|)}{\Gamma(1-d+|n-j|)}\left\{\left(1+\beta_{1}^{2}\right) \alpha_{1}^{|j|}-\beta_{1}\left(\alpha_{1}^{|| j|-1|}+\alpha_{1}^{|j|+1}\right)\right\}}{\sum_{j=-\infty}^{\infty} \frac{\Gamma(d+|-j|)}{\Gamma(1-d+|-j|)}\left\{\left(1+\beta_{1}^{2}\right) \alpha_{1}^{|j|}-\beta_{1}\left(\alpha_{1}^{|| j|-1|}+\alpha_{1}^{|j|+1}\right)\right\}} .
$$


Finally, using the fact that

$$
\sum_{j=0}^{\infty} \frac{\Gamma(d+|n-j|)}{\Gamma(1-d+|n-j|)} \alpha_{1}^{j}=\frac{\Gamma(d+n)}{\Gamma(1-d+n)} F\left(d-n, 1 ; 1-d-n ; \alpha_{1}\right),
$$

we obtain (13)-(14) by straightforward manipulation.

In Figure 1, we plot the theoretical autocorrelation function of a squared $\operatorname{LMGARCH}(1, d, 1)$ process with $\alpha_{1}=0.1, \beta_{1}=0.2$ and $d \in\{0.2,0.3\} .{ }^{1}$ As expected, the autocorrelations decay at a very slow rate, much slower than the geometric rate that is characteristic of weakly stationary GARCH processes.

\subsection{LMGARCH $(p, d, 0)$ Process}

Now consider the $\operatorname{LMGARCH}(p, d, 0)$ process defined via the fractional $\operatorname{ARIMA}(0, d, p)$ equation

$$
\varepsilon_{t}^{2}=\omega+B(L)(1-L)^{-d} v_{t} .
$$

For the process in (16), we have the following result.

Lemma 2 The process $\left\{\varepsilon_{t}^{2}\right\}$ admits the infinite moving-average representation

$$
\varepsilon_{t}^{2}=\omega+\sum_{j=0}^{\infty} \omega_{j} v_{t-j}
$$

where

$$
\omega_{j}=\sum_{k=0}^{j}\left(\begin{array}{c}
-d \\
j-k
\end{array}\right)(-1)^{j-k} \pi_{k}
$$

and

$$
\pi_{k} \triangleq \sum_{r=0}^{\min \{k, p\}}\left(-\beta_{r}\right) \quad\left(\beta_{0} \triangleq-1\right) .
$$

Proof. The desired result is obtained straightforwardly from (16) by using (12).

The autocorrelations of the process defined by (16) are obtained next.

Proposition 2 The autocorrelation function of $\left\{\varepsilon_{t}^{2}\right\}$ is given by

$$
\rho_{n}\left(\varepsilon_{t}^{2}\right)=\gamma_{n} / \gamma_{0}, \quad n \geqslant 1,
$$

where

$$
\gamma_{n}=\frac{\Gamma(1-2 d)}{\Gamma(d) \Gamma(1-d)} \sum_{l=-p}^{p} \Phi_{|l|} \frac{\Gamma(d+n+l)}{\Gamma(1-d+n+l)}, \quad n \geqslant 0
$$

with

$$
\Phi_{|l|} \triangleq \sum_{k=0}^{p-|l|} \beta_{k} \beta_{k+|l|} \quad\left(\beta_{0} \triangleq-1\right) .
$$

\footnotetext{
${ }^{1}$ The Gaussian hypergeometric series was evaluated using Mathematica.
} 
Proof. Using the fact that $\rho_{n}\left(\varepsilon_{t}^{2}\right)=\left(\sum_{j=0}^{\infty} \omega_{j} \omega_{j+n}\right) /\left(\sum_{j=0}^{\infty} \omega_{j}^{2}\right)$, we have in view of (18) that

$$
\rho_{n}\left(\varepsilon_{t}^{2}\right)=\frac{\sum_{l=0}^{p} \Phi_{l} H_{l}\left[\sum_{j=0}^{\infty}\left(\begin{array}{c}
-d \\
j
\end{array}\right)\left(\begin{array}{c}
-d \\
j+|n-l|
\end{array}\right)(-1)^{|n-l|}+\sum_{j=0}^{\infty}\left(\begin{array}{c}
-d \\
j
\end{array}\right)\left(\begin{array}{c}
-d \\
j+n+l
\end{array}\right)(-1)^{n+l}\right]}{\sum_{l=0}^{p} \Phi_{l} H_{l}\left[\sum_{j=0}^{\infty}\left(\begin{array}{c}
-d \\
j
\end{array}\right)\left(\begin{array}{c}
-d \\
j+|-l|
\end{array}\right)(-1)^{|-l|}+\sum_{j=0}^{\infty}\left(\begin{array}{c}
-d \\
j
\end{array}\right)\left(\begin{array}{c}
-d \\
j+l
\end{array}\right)(-1)^{l}\right]},
$$

where

$$
H_{l} \triangleq \begin{cases}\frac{1}{2}, & \text { if } l=0 \\ 1, & \text { if } l \neq 0\end{cases}
$$

Hence, since

$$
\sum_{k=0}^{\infty}\left(\begin{array}{c}
-d \\
|n-l|+k
\end{array}\right)\left(\begin{array}{c}
-d \\
k
\end{array}\right)(-1)^{|n-l|}=\frac{\Gamma(1-2 d) \Gamma(d+|n-l|)}{\Gamma(d) \Gamma(1-d) \Gamma(1-d+|n-l|)}
$$

(19)-(20) follow.

Figure 2 shows the theoretical autocorrelation function of a squared $\operatorname{LMGARCH}(1, d, 0)$ process with $\beta_{1}=0.1$ and $d \in\{0.2,0.3\}$. The shape of the autocorrelation functions is very similar to those for the $\mathrm{LMGARCH}(1, d, 0)$ process, exhibiting a rate of decay much slower than geometric.

\subsection{LMGARCH $(0, d, q)$ Process}

Next consider the $\operatorname{LMGARCH}(0, d, q)$ process defined via the fractional $\operatorname{ARIMA}(q, d, 0)$ equation

$$
\varepsilon_{t}^{2}=\omega+A^{-1}(L)(1-L)^{-d} v_{t},
$$

where it is assumed that the roots of $A(z)=0$ are simple. The moving-average representation of the process in (21) is as follows.

Lemma 3 The process $\left\{\varepsilon_{t}^{2}\right\}$ admits the infinite moving-average representation

$$
\varepsilon_{t}^{2}=\omega+\sum_{j=0}^{\infty} \omega_{j} v_{t-j}
$$

where

$$
\omega_{j}=\sum_{i=1}^{q} \alpha_{i}^{+} \sum_{k=0}^{j}\left(\begin{array}{c}
-d \\
j-k
\end{array}\right) \alpha_{i}^{k}(-1)^{j-k}
$$

and

$$
\alpha_{i}^{+} \triangleq \frac{\alpha_{i}^{q-1}}{\prod_{k=1, k \neq i}^{q}\left(\alpha_{i}-\alpha_{k}\right)} .
$$

Proof. From (21), we have that

$$
\varepsilon_{t}^{2}=\omega+(1-L)^{-d}\left[\prod_{j=1}^{q}\left(1-\alpha_{j} L\right)\right]^{-1} v_{t}
$$


Hence, in view of the fact that

$$
\begin{gathered}
\prod_{j=1}^{q}\left(1-\alpha_{j} L\right)=\sum_{i=1}^{q} \frac{\alpha_{i}^{+}}{1-\alpha_{i} L} \\
(1-L)^{-d}=\sum_{j=0}^{\infty}\left(\begin{array}{c}
-d \\
j
\end{array}\right)(-1)^{j} L^{j}, \quad \text { and } \quad\left(1-\alpha_{i} L\right)^{-1}=\sum_{j=0}^{\infty}\left(\begin{array}{c}
-1 \\
j
\end{array}\right)\left(-\alpha_{i} L\right)^{j},
\end{gathered}
$$

(22)-(23) follow.

The autocorrelation structure of the process defined by (21) is established next.

Proposition 3 The autocorrelation function of $\left\{\varepsilon_{t}^{2}\right\}$ is given by

$$
\rho_{n}\left(\varepsilon_{t}^{2}\right)=\gamma_{n} / \gamma_{0}, \quad n \geqslant 1,
$$

where

$$
\begin{aligned}
\gamma_{n}= & \frac{\Gamma(1-2 d)}{\Gamma(d) \Gamma(1-d)} \sum_{i=1}^{q} \bar{\alpha}_{i}\left\{\frac{\Gamma(d+n)}{\Gamma(1-d+n)} F\left(d+n, 1 ; 1-d+n ; \alpha_{i}\right)\right. \\
& \left.+\alpha_{i} \frac{\Gamma(d+n-1)}{\Gamma(n-d)} F\left(d-n+1,1 ; 2-d-n ; \alpha_{i}\right)\right\}, \quad n \geqslant 0,
\end{aligned}
$$

with

$$
\bar{\alpha}_{i} \triangleq \frac{\alpha_{i}^{+}}{\prod_{k=1}^{q}\left(1-\alpha_{i} \alpha_{k}\right)} .
$$

Proof. In view of (23), we have

$$
\rho_{n}\left(\varepsilon_{t}^{2}\right)=\frac{\sum_{j=0}^{\infty} \omega_{j} \omega_{j+n}}{\sum_{j=0}^{\infty} \omega_{j}^{2}}=\frac{\sum_{k=0}^{\infty} \sum_{j=-\infty}^{\infty} \phi_{n-j}^{k} \eta_{j}}{\sum_{k=0}^{\infty} \sum_{j=-\infty}^{\infty} \phi_{-j}^{k} \eta_{j}},
$$

where

$$
\phi_{n-j}^{k} \triangleq\left(\begin{array}{c}
-d \\
|n-j|+k
\end{array}\right)\left(\begin{array}{c}
-d \\
k
\end{array}\right)(-1)^{|n-j|} \quad \text { and } \quad \eta_{j} \triangleq \sum_{i=1}^{q} \sum_{r=1}^{q} \sum_{m=0}^{\infty} \alpha_{i}^{+} \alpha_{i}^{m} \alpha_{r}^{+} \alpha_{r}^{m+|j|} .
$$

But since

$$
\sum_{k=0}^{\infty} \phi_{n-j}^{k}=\frac{\Gamma(1-2 d) \Gamma(d+|n-j|)}{\Gamma(d) \Gamma(1-d) \Gamma(1-d+|n-j|)} \quad \text { and } \quad \sum_{k=1}^{q} \frac{\alpha_{i}^{+} \alpha_{k}^{+}}{1-\alpha_{i} \alpha_{k}}=\bar{\alpha}_{i},
$$

it follows that

$$
\begin{aligned}
\rho_{n}\left(\varepsilon_{t}^{2}\right) & =\frac{\sum_{j=-\infty}^{\infty} \frac{\Gamma(d+|n-j|)}{\Gamma(1-d+|n-j|)} \sum_{i=1}^{q} \bar{\alpha}_{i} \alpha_{i}^{|j|}}{\sum_{j=-\infty}^{\infty} \frac{\Gamma(d+|-j|)}{\Gamma(1-d+|-j|)} \sum_{i=1}^{q} \bar{\alpha}_{i} \alpha_{i}^{|j|}} \\
& =\frac{\sum_{j=0}^{\infty} \sum_{i=1}^{q} \bar{\alpha}_{i} \alpha_{i}^{j}\left[\frac{\Gamma(d+n+j)}{\Gamma(1-d+n+j)}+\alpha_{i} \frac{\Gamma(d+|n-1-j|)}{\Gamma(1-d+|n-1-j|)}\right]}{\sum_{j=0}^{\infty} \sum_{i=1}^{q} \bar{\alpha}_{i} \alpha_{i}^{j}\left[\frac{\Gamma(d+j)}{\Gamma(1-d+j)}+\alpha_{i} \frac{\Gamma(d+|-1-j|)}{\Gamma(1-d+|-1-j|)}\right]} .
\end{aligned}
$$


Finally, using

$$
\sum_{j=0}^{\infty} \frac{\Gamma(d+n+j)}{\Gamma(1-d+n+j)} \alpha_{i}^{j}=\frac{\Gamma(d+n)}{\Gamma(1-d+n)} F\left(d+n, 1 ; 1-d+n ; \alpha_{i}\right),
$$

we obtain $(24)-(25)$.

Remark. Our results are limited to $\operatorname{LMGARCH}(0, d, q)$ processes for which $\alpha_{i} \neq \alpha_{k}$ for all $i, k \in\{1, \ldots, q\}$ such that $i \neq k$. However, as Sowell (1992) remarked, this might not be an overly restrictive requirement since, in the space of polynomials of a given order, the subset which has repeated zeroes is a set with zero Lebesgue measure.

Figure 3 shows a plot of the theoretical autocorrelation function of a squared $\operatorname{LMGARCH}(0, d, 1)$ process with $\alpha_{1}=0.1$ and $d \in\{0.2,0.3\}$. As before, the autocorrelations decrease extremely slowly.

\subsection{LMGARCH $(p, d, q)$ Process}

We finally consider the general $\operatorname{LMGARCH}(p, d, q)$ process defined via (4)-(5) with the added restriction that the roots of $A(z)=0$ are simple. The moving-average representation of such a process is given in the lemma that follows.

Lemma 4 The process $\left\{\varepsilon_{t}^{2}\right\}$ admits the infinite moving-average representation

$$
\varepsilon_{t}^{2}=\omega+\sum_{j=0}^{\infty} \omega_{j} v_{t-j}
$$

where

$$
\begin{gathered}
\omega_{j}=\sum_{i=1}^{q} \alpha_{i}^{+} \sum_{k=0}^{j}\left(\begin{array}{c}
-d \\
j-k
\end{array}\right) \pi_{i k}(-1)^{j-k} \\
\pi_{i k} \triangleq \sum_{r=0}^{\min \{k, p\}} \alpha_{i}^{k-r}\left(-\beta_{r}\right) \quad\left(\beta_{0} \triangleq-1\right), \quad \text { and } \quad \alpha_{i}^{+} \triangleq \frac{\alpha_{i}^{q-1}}{\prod_{k=1, k \neq i}^{q}\left(\alpha_{i}-\alpha_{k}\right)} .
\end{gathered}
$$

Proof. From (4)-(5), we have that

$$
\varepsilon_{t}^{2}=\omega+(1-L)^{-d}\left[\prod_{j=1}^{q}\left(1-\alpha_{j} L\right)\right]^{-1} B(L) v_{t} .
$$

Hence, on account of

$$
\begin{gathered}
\prod_{j=1}^{q}\left(1-\alpha_{j} L\right)=\sum_{i=1}^{q} \frac{\alpha_{i}^{+}}{1-\alpha_{i} L}, \\
(1-L)^{-d}=\sum_{j=0}^{\infty}\left(\begin{array}{c}
-d \\
j
\end{array}\right)(-1)^{j} L^{j}, \quad \text { and } \quad\left(1-\alpha_{i} L\right)^{-1} B(L)=\sum_{r=0}^{\infty} \pi_{i r} L^{r},
\end{gathered}
$$


(26)-(27) follow.

Next, we establish a representation for the autocorrelation function of the squared values of the $\operatorname{LMGARCH}(p, d, q)$ process.

Theorem 1 The autocorrelation function of $\left\{\varepsilon_{t}^{2}\right\}$ is given by

$$
\rho_{n}\left(\varepsilon_{t}^{2}\right)=\gamma_{n} / \gamma_{0}, \quad n \geqslant 1
$$

where

$$
\begin{gathered}
\gamma_{n}=\sum_{i=1}^{q} \sum_{l=0}^{p} \Phi_{l} \bar{\alpha}_{i} \Theta\left(d, n, l, \alpha_{i}\right), \quad n \geqslant 0, \\
\Phi_{l} \triangleq \sum_{k=0}^{p-l} \beta_{k} \beta_{k+l} \quad\left(\beta_{0} \triangleq-1\right), \quad \bar{\alpha}_{i} \triangleq \frac{\alpha_{i}^{+}}{\prod_{k=1}^{q}\left(1-\alpha_{i} \alpha_{k}\right)}, \\
\Theta\left(d, n, l, \alpha_{i}\right) \triangleq \quad \frac{\Gamma(d+n+l)}{\Gamma(1-d+n+l)} F\left(d+n+l, 1 ; 1-d+n+l ; \alpha_{i}\right) \\
+\mathbf{1}_{l} \frac{\Gamma(d+n-l)}{\Gamma(1-d+n-l)} F\left(d-n+l, 1 ; 1-d-n+l ; \alpha_{i}\right) \\
+\alpha_{i}\left[\mathbf{1}_{l} \frac{\Gamma(d+n+1-l)}{\Gamma(2-d+n-l)} F\left(d+n+1-l, 1 ; 2-d+n-l ; \alpha_{i}\right)\right. \\
\left.+\frac{\Gamma(d+n-1+l)}{\Gamma(n-d+l)} F\left(d-n+1-l, 1 ; 2-d-n-l ; \alpha_{i}\right)\right],
\end{gathered}
$$

and

$$
\mathbf{1}_{l} \triangleq \begin{cases}0, & \text { if } l=0 \\ 1, & \text { if } l \neq 0\end{cases}
$$

Proof. In view of (27), we have

$$
\rho_{n}\left(\varepsilon_{t}^{2}\right)=\frac{\sum_{j=0}^{\infty} \omega_{j} \omega_{j+n}}{\sum_{j=0}^{\infty} \omega_{j}^{2}}=\frac{\sum_{k=0}^{\infty} \sum_{j=-\infty}^{\infty} \phi_{n-j}^{k} \eta_{j}}{\sum_{k=0}^{\infty} \sum_{j=-\infty}^{\infty} \phi_{-j}^{k} \eta_{j}},
$$

where

$$
\phi_{n-j}^{k} \triangleq\left(\begin{array}{c}
-d \\
|n-j|+k
\end{array}\right)\left(\begin{array}{c}
-d \\
k
\end{array}\right)(-1)^{|n-j|}, \quad \text { and } \quad \eta_{j} \triangleq \sum_{i=1}^{q} \sum_{s=1}^{q} \sum_{m=0}^{\infty} \alpha_{i}^{+} \alpha_{s}^{+} \pi_{i m} \pi_{s, m+|j|} .
$$

But since

$$
\sum_{k=0}^{\infty} \phi_{n-j}^{k}=\frac{\Gamma(1-2 d) \Gamma(d+|n-j|)}{\Gamma(d) \Gamma(1-d) \Gamma(1-d+|n-j|)}, \quad \text { and } \quad \sum_{k=1}^{q} \frac{\alpha_{i}^{+} \alpha_{k}^{+}}{1-\alpha_{i} \alpha_{k}}=\bar{\alpha}_{i}
$$


it follows that

$$
\begin{aligned}
\rho_{n}\left(\varepsilon_{t}^{2}\right)= & \frac{\sum_{j=-\infty}^{\infty} \frac{\Gamma(d+|n-j|)}{\Gamma(1-d+|n-j|)} \sum_{i=1}^{q} \sum_{l=0}^{p} \Phi_{l} \bar{\alpha}_{i}\left(\mathbf{1}_{l} \alpha_{i}^{|| j|-l|}+\alpha_{i}^{|j|+l}\right)}{\sum_{j=-\infty}^{\infty} \frac{\Gamma(d+|-j|)}{\Gamma(1-d+|-j|)} \sum_{i=1}^{q} \sum_{l=0}^{p} \Phi_{l} \bar{\alpha}_{i}\left(\mathbf{1}_{l} \alpha_{i}^{|| j|-l|}+\alpha_{i}^{|j|+l}\right)}, \\
= & \left\{\sum _ { l = 0 } ^ { p } \sum _ { j = 0 } ^ { \infty } \Phi _ { l } \sum _ { i = 1 } ^ { q } \overline { \alpha } _ { i } \left[\frac{\mathbf{1}_{l} \Gamma(d+|n-l-j|)}{\Gamma(1-d+|n-l-j|)}+\frac{\Gamma(d+n+l+j)}{\Gamma(1-d+n+l+j)}\right.\right. \\
& \left.\left.+\left(\frac{\Gamma(d+|n-1+l-j|)}{\Gamma(1-d+|n-1+l-j|)}+\frac{\mathbf{1}_{l} \Gamma(d+|n+1-l+j|)}{\Gamma(1-d+|n+1-l+j|)}\right) \alpha_{i}\right] \alpha_{i}^{j}\right\} \\
& \times\left\{\sum _ { l = 0 } ^ { p } \sum _ { j = 0 } ^ { \infty } \Phi _ { l } \sum _ { i = 1 } ^ { q } \overline { \alpha } _ { i } \left[\frac{\mathbf{1}_{l} \Gamma(d+|-l-j|)}{\Gamma(1-d+|-l-j|)}+\frac{\Gamma(d+l+j)}{\Gamma(1-d+l+j)}\right.\right. \\
& \left.\left.+\left(\frac{\Gamma(d+|-1+l-j|)}{\Gamma(1-d+|-1+l-j|)}+\frac{\mathbf{1}_{l} \Gamma(d+|1-l+j|)}{\Gamma(1-d+|1+j-l|)}\right) \alpha_{i}\right] \alpha_{i}^{j}\right\}^{-1} .
\end{aligned}
$$

Hence, upon observing that

$$
\sum_{j=0}^{\infty} \frac{\Gamma(d+n+l+j)}{\Gamma(1-d+n+l+j)} \alpha_{i}^{j}=\frac{\Gamma(d+n+l)}{\Gamma(1-d+n+l)} F\left(d+n+l, 1 ; 1-d+n+l ; \alpha_{i}\right),
$$

(28)-(29) are obtained.

\section{An Empirical Illustration}

As an empirical illustration, we examine the properties of continuously compounded daily rates of return for the Deutschmark exchange rate vis-à-vis the US Dollar over the period from $31 / 10 / 1983$ to $31 / 12 / 1992$ (2,394 observations in total). To evaluate how well the secondorder properties of the squared exchange rate returns are approximated by various models, we compared the sample autocorrelation function of the squared returns with the theoretical autocorrelation functions of $\operatorname{LMGARCH}(1, d, 0), \operatorname{LMGARCH}(0, d, 1), \operatorname{LMGARCH}(1, d, 1)$, and $\operatorname{IGARCH}(1,1)$ processes. The theoretical autocorrelations were evaluated using the formulae given in Propositions 1-3 and the quasi-maximum likelihood parameter estimates reported in Table 1 (obtained under the assumption of conditional Gaussianity). In the $\operatorname{IGARCH}(1,1)$ case, we used the formula for the approximate autocorrelation function given in Ding and Granger (1996).

Figure 4 plots the theoretical and sample autocorrelations for lags up to 2,392. ${ }^{2}$ Evidently, the $\operatorname{LMGARCH}(1, d, 0)$ and $\operatorname{LMGARCH}(0, d, 1)$ processes give the best approximation to the autocorrelations of the squared exchange rate returns. The $\operatorname{LMGARCH}(1, d, 1)$ process has autocorrelations considerable larger than the sample autocorrelations of the returns. The $\operatorname{IGARCH}(1,1)$ process, on the other hand, provides a very bad approximation to the first 295 sample autocorrelations of the returns but is almost as good as the $\operatorname{LMGARCH}(1, d, 0)$ and $\operatorname{LMGARCH}(0, d, 1)$ from then onwards.

\footnotetext{
${ }^{2}$ The quasi-maximum likelihood estimates in Table 1 are such that $0<\sum_{j=0}^{\infty} \omega_{j}^{2}<\frac{3}{2}$ for all three LMGARCH models.
} 
Table 1. Quasi-Maximum Likelihood Estimates ${ }^{\mathrm{a}}$

\begin{tabular}{lccc}
\hline \hline & $(1, d, 0)$ & $(0, d, 1)$ & $(1, d, 1)$ \\
\hline$d$ & $0.2326(0.0365)$ & $0.1847(0.0237)$ & $0.3805(0.0680)$ \\
$\alpha_{1}$ & - & $-0.1260(0.0306)$ & $0.2742(0.0471)$ \\
$\beta_{1}$ & $0.1973(0.0460)$ & - & $0.6114(0.0620)$ \\
\hline
\end{tabular}

${ }^{\mathrm{a}}$ Figures in parentheses are asymptotic standard errors.

\section{Conclusion}

In this paper, we have examined the dependence structure of long-memory autoregressive conditionally heteroscedastic processes. More specifically, we have obtained characterizations of the theoretical autocorrelation function of the squared values of LMGARCH processes. Such processes have been found to describe well the observed autocorrelation structure of many reallife financial time series and are thus of much interest. With our results, one can establish what a fitted model implies about the second-order structure of the squared observations and the extend to which these characteristics are consistent with the correlogram of the data. This is illustrated in an empirical application involving foreign exchange rate data.

\section{References}

[1] Baillie, R. T., Bollerslev, T., and Mikkelsen, H. O. (1996), Fractionally integrated generalized autoregressive conditional heteroskedasticity, Journal of Econometrics, 74, 3-30.

[2] Bollerslev, T. (1986), Generalized autoregressive conditional heteroskedasticity, Journal of Econometrics, 31, 307-327.

[3] Bollerslev, T., and Mikkelsen, H. O. (1996), Modelling and pricing long memory in stock market volatility, Journal of Econometrics, 73, 151-184.

[4] Breidt, F. J., Crato, N., and de Lima, P. (1998), On the detection and estimation of long memory in stochastic volatility, Journal of Econometrics, 83, 325-348.

[5] Dacorogna, M. M., Muller, U. A., Nagler, R. J., Olsen, R. B., and Pictet, O. V. (1993), A geographical model for the daily and weekly seasonal volatility in the foreign exchange market, Journal of International Money and Finance, 12, 413-438.

[6] Ding, Z., and Granger, C. W. J. (1996), Modeling volatility persistence of speculative returns: a new approach, Journal of Econometrics, 73, 185-215.

[7] Ding, Z., Granger, C.W. J., and Engle, R.F. (1993), A long memory property of stock market returns and a new model, Journal of Empirical Finance, 1, 83-106. 
[8] Giraitis, L., Kokoszka, P., and Leipus R. (2000), Stationary ARCH models: dependence structure and central limit theorem, Econometric Theory, 16, 3-22.

[9] Henry, M. (2001), Averaged periodogram spectral estimation with long-memory conditional heteroscedasticity, Journal of Time Series Analysis, 22, 431-459.

[10] Robinson, P. M. (1991), Testing for strong serial correlation and dynamic conditional heteroscedasticity in multiple regression, Journal of Econometrics, 47, 67-84.

[11] Robinson, P. M., and Henry, M. (1999), Long and short memory conditional heteroskedasticity in estimating the memory parameter of levels, Econometric Theory, 15, 299-336.

[12] Robinson, P. M, and Zaffaroni, P. (1997), Modelling nonlinearity and long memory in time series, in C. D. Cutler and D. T. Kaplan (eds.), Nonlinear Dynamics and Time Series, Fields Institute Communications Vol. 11, Providence: American Mathematical Society, pp. 161170 .

[13] Sowell, F. (1992), Maximum likelihood estimation of stationary univariate fractionally integrated time series models, Journal of Econometrics, 53, 165-188.

[14] Zaffaroni, P. (2000), Stationarity and memory of $\mathrm{ARCH}(\infty)$ models, unpublished manuscript, Bank of Italy. 


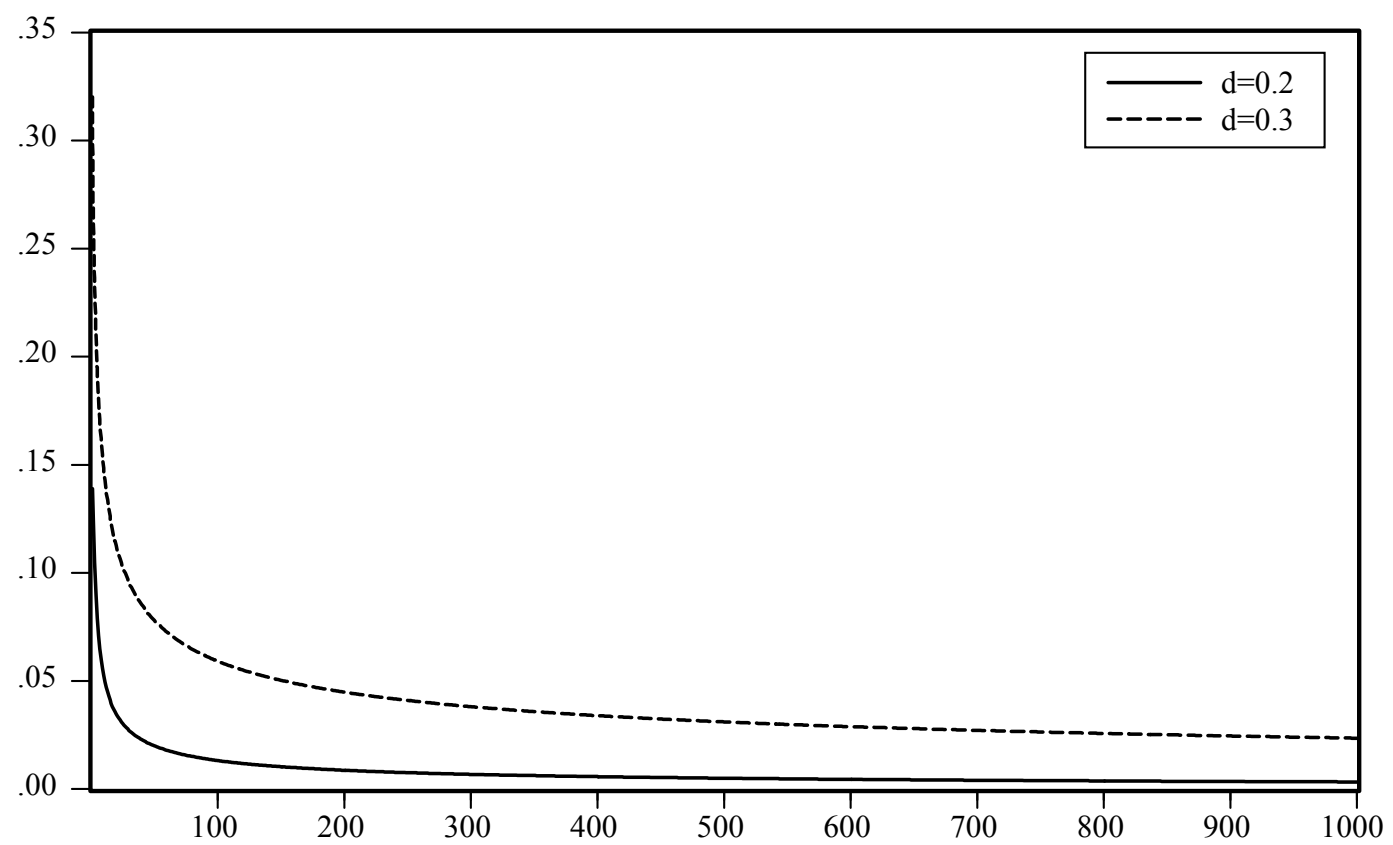

Figure 1: Autocorrelation function of squared $\operatorname{LMGARCH}(1, d, 1)$ process.

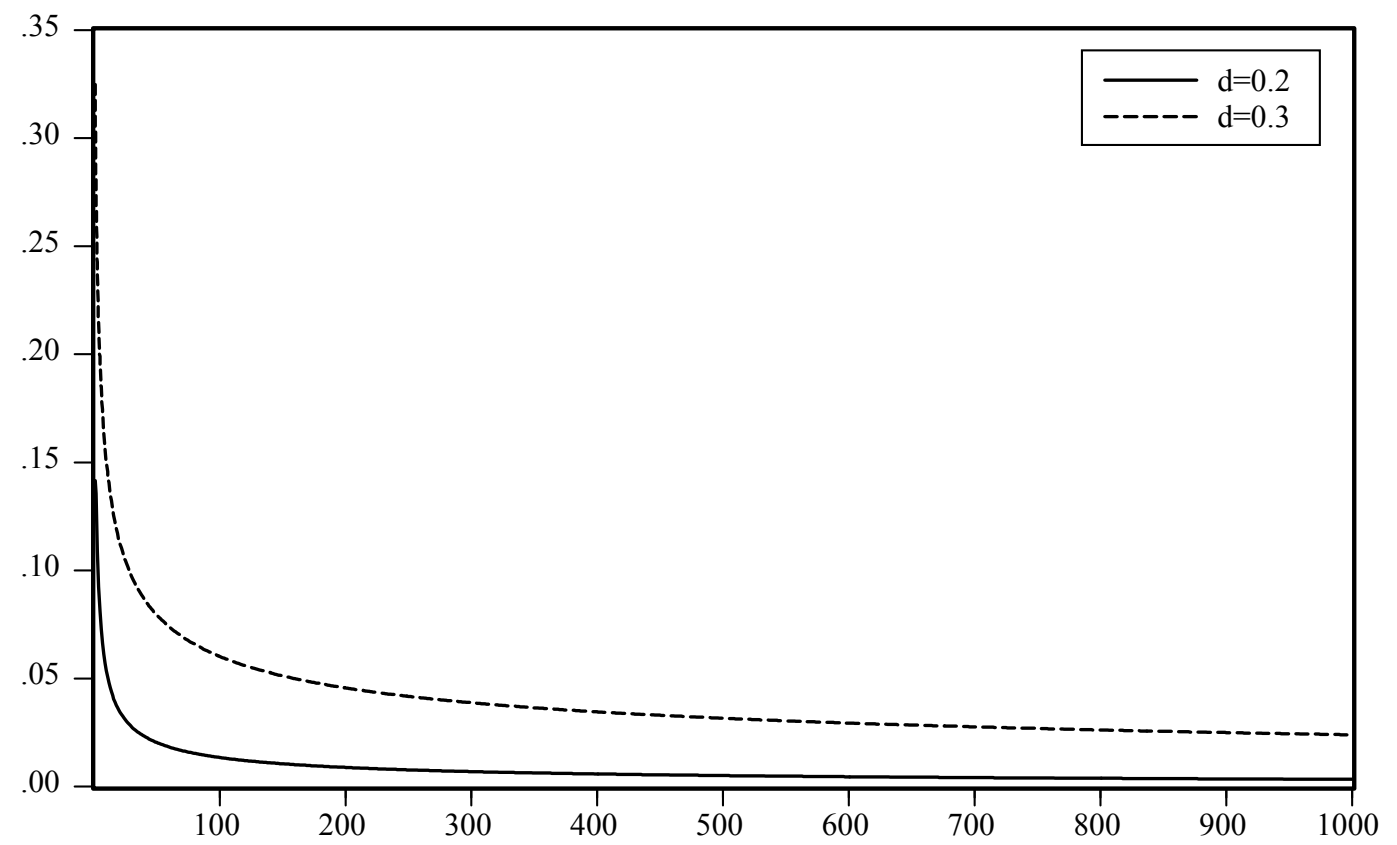

Figure 2: Autocorrelation function of squared LMGARCH $(1, d, 0)$ process. 


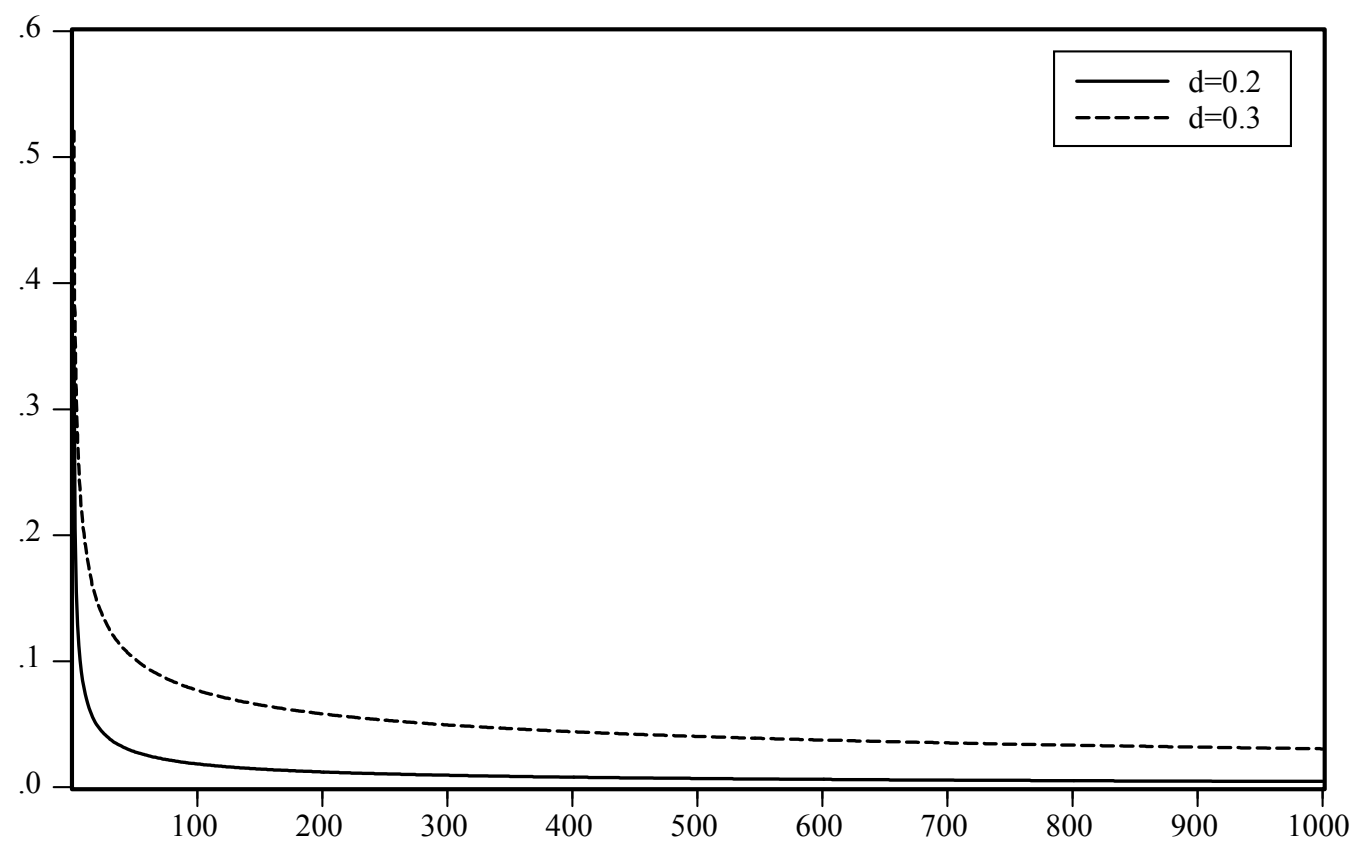

Figure 3: Autocorrelation function of squared $\operatorname{LMGARCH}(0, d, 1)$ process.

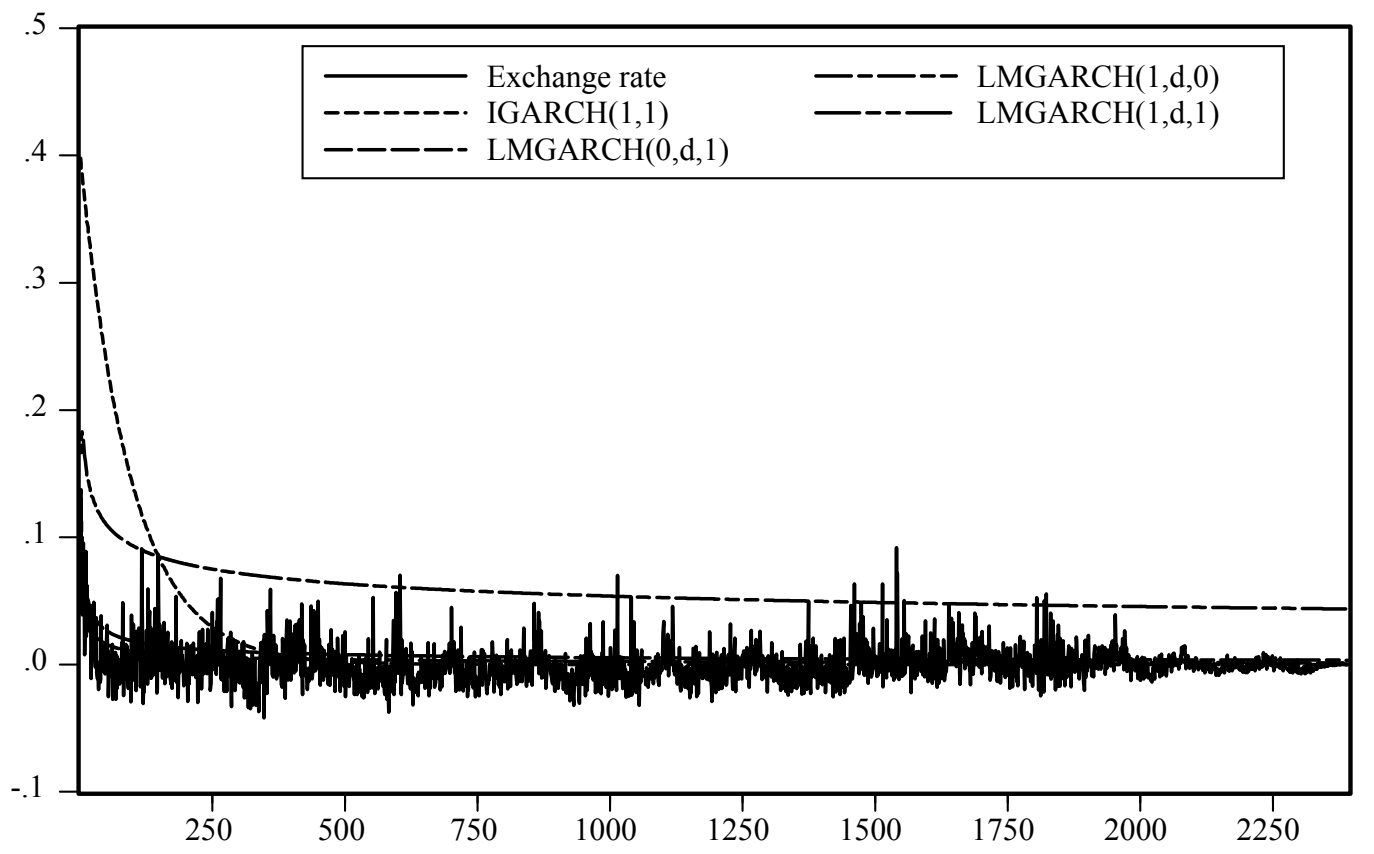

Figure 4: Sample autocorrelations of squared exchange-rate returns and theoretical LMGARCH autocorrelation functions. 